Kragujevac Journal of Mathematics

Volume 44(2) (2020), Pages 273-286.

\title{
SOME MATRIX AND COMPACT OPERATORS OF THE ABSOLUTE FIBONACCI SERIES SPACES
}

\author{
FADIME GÖKÇE ${ }^{1}$ AND MEHMET ALI SARIGÖL ${ }^{1}$
}

\begin{abstract}
In the present paper, we introduce the absolute Fibonacci space $\left|F_{u}\right|_{k}$, give some inclusion relations and investigate topological and algebraic structure such as $B K$-space, $\alpha-, \beta-, \gamma$ - duals and Schauder basis. Further, we characterize certain matrix and compact operators on these spaces, also determine their norms and Hausdroff meausures of noncompactness.
\end{abstract}

\section{INTRODUCTION}

Let $\omega$ be the set of all sequences of complex numbers. We write $c, \ell_{\infty}, c_{s}, b_{s}$ and $\ell_{k}, k \geq 1$, for the sequence space of all convergent, bounded sequences; for the spaces of all convergent, bounded, $k$-absolutely convergent series, respectively. Let $X$ and $Y$ be two subspaces of $\omega$ and $A=\left(a_{n v}\right)$ be an arbitrary infinite matrix of complex numbers. If the series

$$
A_{n}(x)=\sum_{v=0}^{\infty} a_{n v} x_{v}
$$

converges for all $n \in \mathbb{N}=\{0,1,2, \ldots\}$, then, by $A(x)=\left(A_{n}(x)\right)$, we denote the $A$ transform of the sequence $x=\left(x_{v}\right)$. Also, we say that $A$ defines a matrix transformation from $X$ into $Y$, and denote it by $A \in(X, Y)$ or $A: X \rightarrow Y$ if $A x=\left(A_{n}(x)\right) \in Y$ for every $x \in X$. The $\alpha$-, $\beta$-, $\gamma$ - duals of $X$ and the domain of the matrix $A$ in $X$ are defined by

$$
\begin{aligned}
& X^{\alpha}=\left\{\epsilon \in \omega:\left(\epsilon_{n} x_{n}\right) \in \ell \text { for all } x \in X\right\}, \\
& X^{\beta}=\left\{\epsilon \in \omega:\left(\epsilon_{n} x_{n}\right) \in c_{s} \text { for all } x \in X\right\}, \\
& X^{\gamma}=\left\{\epsilon \in \omega:\left(\epsilon_{n} x_{n}\right) \in b_{s} \text { for all } x \in X\right\}
\end{aligned}
$$

Key words and phrases. Absolute summability, Fibonacci numbers, matrix transformations, sequence spaces, bounded operators, Hausdroff meausures of noncompactness.

2010 Mathematics Subject Classification. Primary: 40C05, 11B39, 40D25, 40F05, 46A45.

DOI 10.46793/KgJMat2002.273G

Received: December 21, 2017.

Accepted: April 09, 2018. 
and

$$
X_{A}=\left\{x=\left(x_{n}\right) \in \omega: A(x) \in X\right\},
$$

respectively. Further, $X$ is said to be a $B K$-space if it is a complete normed space with continuous coordinates $p_{n}: X \rightarrow \mathbb{C}$ defined by $p_{n}(x)=x_{n}$ for all $n \in \mathbb{N}$. If there exists unique sequence of coefficients $\left(x_{k}\right)$ such that, for each $x \in X$,

$$
\left\|x-\sum_{k=0}^{m} x_{k} b_{k}\right\| \rightarrow 0, \quad m \rightarrow \infty,
$$

then, the sequence $\left(b_{k}\right)$ is called the Schauder basis (or briefly basis) for a normed sequence space $X$, and in this case we write $x=\sum_{k=0}^{\infty} x_{k} b_{k}$. For instance, the sequence $\left(e^{(j)}\right)$ is the Schauder basis of the space $\ell_{k}$, where $e^{(j)}$ is the sequence whose only nonzero term is 1 in $j$ th place for each $j \in \mathbb{N}$.

Now take $\sum x_{v}$ as an infinite series with $n$th partial sum $s_{n}$ and let $\left(u_{n}\right)$ be a sequence of positive terms. Then, the series $\sum x_{v}$ is said to be summable $\left|A, u_{n}\right|_{k}$, $k \geq 1$, if (see [32])

$$
\sum_{n=0}^{\infty} u_{n}^{k-1}\left|\Delta A_{n}(s)\right|^{k}<\infty
$$

where $\Delta A_{n}(s)=A_{n}(s)-A_{n-1}(s), A_{-1}(s)=0$.

Note that this method includes some well known methods. For example, if $A$ is the matrix of weighted mean $\left(\bar{N}, p_{n}\right)$ (resp. $\left.u_{n}=P_{n} / p_{n}\right)$, then it reduces to the summability $\left|\bar{N}, p_{n}, u_{n}\right|_{k}[36]$ (the summability $\left|\bar{N}, p_{n}\right|_{k}[10]$ ). Also if we take $A$ as the matrix of Cesàro mean of order $\alpha>-1$ and $u_{n}=n$, then we get summability $|C, \alpha|_{k}$ in Flett's notation [11].

A large literature has recently grown up, concerned with producing sequence spaces by means of matrix domain of a special limitation method and studying their algebraic, topological structure and matrix transformations (see $[1-7,15-18,25]$ ). Also, some series spaces have been derived and studied by absolute summability methods from a different point of view (see [9-14,23-26,28-34,36]). The aim of this paper is to define the space $\left|F_{u}\right|_{k}$ combining absolute summability and Fibonacci matrix given by Kara [15], investigate some inclusion relation, construct their $\alpha-, \beta-, \gamma-$ duals, basis and characterize some matrix operators related to that space, and also determine their norms and Hausdroff measures of noncompactness.

Firstly, we mention some properties of Fibonacci numbers as follows: the sequence $\left(f_{n}\right)$ of Fibonacci numbers is given by the relations

$$
f_{0}=f_{1}=1 \text { and } f_{n}=f_{n-1}+f_{n-2} \text { for } n \geq 2
$$

that is, each term is equal to the sum of the previous two terms. The sequences of Fibonacci numbers have been important for artist, architects, physicists and mathematicians since the old. The ratio of Fibonacci numbers converges to the golden ratio which is one of the most interesting irrationals having an important role in number 
theory, algorithms, network theory, etc. Also, Fibonacci numbers have the following properties [19]:

$$
\begin{gathered}
\sum_{n} \frac{1}{f_{n}} \text { converges }, \\
f_{n-1}^{2}+f_{n} f_{n-1}-f_{n}^{2}=(-1)^{n+1}, \quad n \geq 1, \\
\lim _{n \rightarrow \infty} \frac{f_{n+1}}{f_{n}}=\frac{1+\sqrt{5}}{2}=1.61803398875 \ldots
\end{gathered}
$$

Fibonacci matrix $F=\left(\hat{f}_{n v}\right)$ has recently been defined by Kara [15] as follows:

$$
\hat{f}_{n v}= \begin{cases}\frac{-f_{n+1}}{f_{n}}, & v=n-1, \\ \frac{f_{n}}{f_{n+1}}, & v=n, \\ 0, & v>n \text { or } 0 \leq v<n-1,\end{cases}
$$

where $f_{n}$ be the $n$th Fibonacci number for every $n \in \mathbb{N}$. Note that if we take the Fibonacci matrix instead of $A$, then $\left|A, u_{n}\right|_{k}$ summability reduces to the absolute Fibonacci summability. On the other hand, since $\left(s_{n}\right)$ is a sequence of partial sum of the series $\sum x_{v}$, we get

$$
A_{n}(s)=\sum_{v=0}^{n} \hat{f}_{n v} s_{v}=\sum_{j=0}^{n} x_{j} \sum_{v=j}^{n} \hat{f}_{n v}=x_{n} \hat{f}_{n n}+\sum_{j=0}^{n-1}\left(\hat{f}_{n n}+\hat{f}_{n, n-1}\right) x_{j}
$$

and so,

$$
\begin{aligned}
\Delta A_{n}(s) & =x_{n} \frac{f_{n}}{f_{n+1}}+x_{n-1}\left(\frac{(-1)^{n}}{f_{n} f_{n+1}}-\frac{f_{n+1}}{f_{n}}\right)+\sum_{j=0}^{n-2}(-1)^{n} \frac{f_{n-1}+f_{n+1}}{f_{n-1} f_{n} f_{n+1}} x_{j} \\
& =\sum_{j=0}^{n} \sigma_{n j} x_{j},
\end{aligned}
$$

where

$$
\sigma_{n j}= \begin{cases}\frac{f_{n}}{f_{n+1}}, & j=n, \\ \frac{(-1)^{n}}{f_{n} f_{n+1}}-\frac{f_{n+1}}{f_{n}}, & j=n-1, \\ (-1)^{n} \frac{f_{n-1}+f_{n+1}}{f_{n-1} f_{n} f_{n+1}}, & 0 \leq j \leq n-2, \\ 0, & j>n .\end{cases}
$$

Now, we introduce the absolute Fibonacci space as follows:

$$
\left|F_{u}\right|_{k}=\left\{x \in \omega: \sum_{n=0}^{\infty} u_{n}^{k-1}\left|\sum_{j=0}^{n} \sigma_{n j} x_{j}\right|^{k}<\infty\right\} .
$$

Also, it may be written that

$$
\left(E^{(k)} \circ T\right)_{n}(x)=u_{n}^{1 / k^{*}}\left(T_{n}(x)-T_{n-1}(x)\right),
$$


where

$$
\begin{aligned}
& t_{n v}= \begin{cases}\frac{f_{n}}{f_{n+1}}, & v=n, \\
\frac{f_{n}^{2}-f_{n+1}^{2}}{f_{n} f_{n+1}}, & 0 \leq v \leq n-1, \\
0, & v>n,\end{cases} \\
& e_{n v}^{(k)}= \begin{cases}u_{n}^{1 / k^{*}}, & v=n, \\
-u_{n}^{1 / k^{*}}, & v=n-1, \\
0, & v \neq n, n-1,\end{cases}
\end{aligned}
$$

and $k^{*}$ is the conjugate of $k$, i.e., $1 / k+1 / k^{*}=1$ for $k>1$, and $1 / k^{*}=0$ for $k=1$. With these matrices $T=\left(t_{n v}\right)$ and $E^{(k)}=\left(e_{n v}^{(k)}\right)$, according to the notation (1.1), it is obvious that $\left|F_{u}\right|_{k}=\left(\ell_{k}\right)_{E^{(k)} \circ T}$. Further, since every triangle matrix has a unique inverse which also is a triangle [37], $T$ and $E^{(k)}$ have a unique inverse $\tilde{T}=\left(\tilde{t}_{n v}\right)$ and $\tilde{E}^{(k)}=\left(\tilde{e}_{n v}\right)$ given by

$$
\begin{gathered}
\tilde{t}_{n v}= \begin{cases}\frac{f_{n+1}}{f_{n}}, & v=n, \\
\frac{f_{n+1}^{2}-f_{n}^{2}}{f_{v} f_{v+1}}, & 0 \leq v \leq n-1, \\
0, & v>n,\end{cases} \\
\tilde{e}_{n v}^{(k)}= \begin{cases}u_{v}^{-1 / k^{*}}, & 0 \leq v \leq n, \\
0, & v>n,\end{cases}
\end{gathered}
$$

respectively.

Before the main theorems, we point out some well known lemmas which are needed in the proofs of theorems.

Lemma 1.1 ([35]). Let $1<k<\infty$. Then, $A \in\left(\ell_{k}, \ell\right)$ if and only if

$$
\|A\|_{\left(\ell_{k}, \ell\right)}=\sup _{N \in \mathfrak{F}}\left\{\sum_{v=0}^{\infty}\left|\sum_{n=0}^{\infty} a_{n v}\right|^{k^{*}}\right\}^{1 / k^{*}},
$$

where $\mathfrak{F}$ denotes the collection of all finite subsets of $\mathbb{N}$.

Lemma 1.1 exposes a rather difficult condition to apply in applications. So the following lemma is more useful in many cases, which gives equivalent norm.

Lemma $1.2([29])$. Let $1<k<\infty$. Then, $A \in\left(\ell_{k}, \ell\right)$ if and only if

$$
\|A\|_{\left(\ell_{k}, \ell\right)}^{\prime}=\left\{\sum_{v=0}^{\infty}\left(\sum_{n=0}^{\infty}\left|a_{n v}\right|\right)^{k^{*}}\right\}^{1 / k^{*}}<\infty .
$$

Moreover, since

$$
\|A\|_{\left(\ell_{k}, \ell\right)} \leq\|A\|_{\left(\ell_{k}, \ell\right)}^{\prime} \leq 4\|A\|_{\left(\ell_{k}, \ell\right)},
$$

there exists $1 \leq \xi \leq 4$ such that $\|A\|_{\left(\ell_{k}, \ell\right)}^{\prime}=\xi\|A\|_{\left(\ell_{k}, \ell\right)}$. 
Lemma $1.3([20])$. Let $1 \leq k<\infty$. Then, $A \in\left(\ell, \ell_{k}\right)$ if and only if

$$
\|A\|_{\left(\ell, \ell_{k}\right)}=\sup _{v}\left\{\sum_{n=0}^{\infty}\left|a_{n v}\right|^{k}\right\}^{\frac{1}{k}} \text {. }
$$

Lemma 1.4 ([35]).

(a) $A \in(\ell, c) \Leftrightarrow(i) \lim _{n} a_{n v}$ exists for $v \geq 0$, (ii) $\sup _{n, v}\left|a_{n v}\right|<\infty$;

(b) $A \in\left(\ell, \ell_{\infty}\right) \Leftrightarrow$ (ii) holds;

(c) If $1<k<\infty$, then, $A \in\left(\ell_{k}, c\right) \Leftrightarrow(i)$ holds, (iii) $\sup _{n} \sum_{v=0}^{\infty}\left|a_{n v}\right|^{k^{*}}<\infty$;

(d) If $1<k<\infty$, then, $A \in\left(\ell_{k}, \ell_{\infty}\right) \Leftrightarrow$ (iii) holds.

\section{The Hausdorff Measure of Noncompactness}

If $S$ and $H$ are subsets of a metric space $(X, d)$ and, for every $h \in H$, there exists an $s \in S$ such that $d(h, s)<\varepsilon$ then, $S$ is called an $\varepsilon$-net of $H$; if $S$ is finite, then the $\varepsilon$-net $S$ of $H$ is called a finite $\varepsilon$-net of $H$. Let $X$ and $Y$ be Banach spaces. A linear operator $L: X \rightarrow Y$ is called compact if its domain is all of $X$ and, for every bounded sequence $\left(x_{n}\right)$ in $X$, the sequence $\left(L\left(x_{n}\right)\right)$ has a convergent subsequence in $Y$. We denote the class of such operators by $\mathrm{C}(X, Y)$. If $Q$ is a bounded subset of the metric space $X$, then the Hausdorff measure of noncompactness of $Q$ is defined by

$$
\chi(Q)=\inf \{\varepsilon>0: Q \text { has a finite } \varepsilon-\text { net in } X\},
$$

and $\chi$ is called the Hausdorff measure of noncompactness.

The following lemma is very important to calculate the Hausdorff measure of noncompactness of a bounded subset of the space $\ell_{k}$.

Lemma $2.1([27])$. Let $Q$ be a bounded subset of the normed space $X$ where $X=\ell_{k}$ for $1 \leq k<\infty$ or $X=c_{0}$. If $P_{n}: X \rightarrow X$ is the operator defined by $P_{n}(x)=$ $\left(x_{0}, x_{1}, \ldots, x_{n}, 0,0, \ldots\right)$ for all $x \in X$, then

$$
\chi(Q)=\lim _{r \rightarrow \infty}\left(\sup _{x \in Q}\left\|\left(I-P_{r}\right)(x)\right\|\right) .
$$

Let $X$ and $Y$ be Banach space and $\chi_{1}$ and $\chi_{2}$ be Hausdorff measures on $X$ and $Y$, the linear operator $L: X \rightarrow Y$ is said to be $\left(\chi_{1}, \chi_{2}\right)$ - bounded if $L(Q)$ is a bounded subset of $Y$ and there exists a positive constant $M$ such that $\chi_{2}(L(Q)) \leq M \chi_{1}(L(Q))$ for every bounded subset $Q$ of $X$. If an operator $L$ is $\left(\chi_{1}, \chi_{2}\right)$ - bounded, then the number

$$
\|L\|_{\left(\chi_{1}, \chi_{2}\right)}=\inf \left\{M>0: \chi_{2}(L(Q)) \leq M \chi_{1}(L(Q)) \text { for all bounded set } Q \subset X\right\}
$$

is called the $\left(\chi_{1}, \chi_{2}\right)$-measure noncompactness of $L$. In particular, if $\chi_{1}=\chi_{2}=\chi$ then we write $\|L\|_{(\chi, \chi)}=\|L\|_{\chi}$. 
Lemma 2.2 ([22]). Let $X$ and $Y$ be Banach spaces and $L \in \mathcal{B}(X, Y)$. Also $S_{x}=$ $\{x \in X:\|x\| \leq 1\}$ be the unit sphere in $X$. Then,

$$
\|L\|_{\chi}=\chi\left(L\left(S_{x}\right)\right)
$$

and

$$
L \in \mathcal{C}(X, Y) \Leftrightarrow\|L\|_{\chi}=0 .
$$

Lemma $2.3([21])$. Let $X$ be a normed sequence space, $T=\left(t_{n v}\right)$ be an infinite triangle matrix, $\chi_{T}$ and $\chi$ denote the Hausdroff measures of noncompactness on $M_{X_{T}}$ and $M_{X}$, the collections of all bounded sets in $X_{T}$ and $X$, respectively. Then, $\chi_{T}(Q)=\chi(T(Q))$ for all $Q \in M_{X_{T}}$.

\section{Absolute Fibonacci Space $\left|F_{u}\right|_{k}$}

In this section, we investigate some inclusion relations, topological and algebraic structures of the space $\left|F_{u}\right|_{k}$. Also we characterize some classes of compact matrix operators on that space and compute their norms and Hausdroff measure of noncompactness.

Firstly, since $\left|F_{u}\right|_{k}$ is generated from $\ell_{k}$, to explain a relation between the spaces $\ell_{k}$ and $\left|F_{u}\right|_{k}$, we begin with the following theorem.

Theorem 3.1. Let $u=\left(u_{n}\right) \in \ell_{\infty}$ and $1 \leq k<\infty$. Then, $\ell_{k} \subset\left|F_{u}\right|_{k}$.

Proof. To prove the inclusion $\ell_{k} \subset\left|F_{u}\right|_{k}$, it is sufficient to show that

$$
\|x\|_{\left|F_{u}\right|_{k}} \leq O(1)\|x\|_{\ell_{k}}
$$

for all $x \in \ell_{k}$. The proof is clear for the case $k=1$, and so it is omitted. Let $k>1$. Then, since the series $\sum_{n} \frac{1}{f_{n}}$ is convergent and $\left(\frac{1}{f_{n}}\right)$ is decreasing sequence, it follows from Abel's Theorem, $\frac{n}{f_{n}} \rightarrow 0$ as $n \rightarrow \infty, \sum_{v=0}^{n}\left|\sigma_{n v}\right|=O(1)$ and $\sum_{n=v}^{\infty}\left|\sigma_{n v}\right|=O(1)$. Now applying Hölder's inequality, we get

$$
\begin{aligned}
\|x\|_{\left|F_{u}\right|_{k}} & =\left\{\sum_{n=0}^{\infty} u_{n}^{k-1}\left|\sum_{v=0}^{n} \sigma_{n v} x_{v}\right|^{k}\right\}^{1 / k} \\
& \leq\left\{\sum_{n=0}^{\infty} u_{n}^{k-1} \sum_{v=0}^{n}\left|\sigma_{n v}\right|\left|x_{v}\right|^{k}\left(\sum_{v=0}^{n}\left|\sigma_{n v}\right|\right)^{k / k^{*}}\right\}^{1 / k} \\
& =O(1)\left\{\sum_{v=0}^{\infty}\left|x_{v}\right|^{k} \sum_{n=v}^{\infty}\left|\sigma_{n v}\right|\right\}^{1 / k} \\
& =O(1)\left\{\sum_{v=0}^{\infty}\left|x_{v}\right|^{k}\right\}^{1 / k}=O(1)\|x\|_{\ell_{k}}
\end{aligned}
$$

which completes the proof. 
Theorem 3.2. Let $1 \leq k \leq q<\infty$. If there is a constant $M>0$ such that $u_{n} \leq M$ for all $n \in \mathbb{N}$, then $\left|F_{u}\right|_{k} \subset\left|F_{u}\right|_{q}$.

Proof. Take $x \in\left|F_{u}\right|_{k}$. Since $\ell_{k} \subset \ell_{q}$, then $\left(u_{n}^{\frac{1}{k^{*}}} \sum_{j=0}^{n} \sigma_{n j} x_{j}\right) \in \ell_{q}$ and also, since $u_{n} \leq M$ for all $n \in \mathbb{N}$,

$$
M^{\frac{q}{k^{*}}-\frac{q}{q^{*}}}\left|u_{n}^{\frac{1}{q^{*}}} \sum_{j=0}^{n} \sigma_{n j} x_{j}\right|^{q} \leq\left|u_{n}^{1 / k^{*}} \sum_{j=0}^{n} \sigma_{n j} x_{j}\right|^{q},
$$

where $k^{*}$ and $q^{*}$ are the conjugate of exponent of $k$ and $q$, respectively. So this gives that $x \in\left|F_{u}\right|_{q}$, which completes the proof.

Theorem 3.3. Let $1 \leq k<\infty$. Then, $\left|F_{u}\right|_{k}$ is BK-space with respect to the norm

$$
\|x\|_{\left|F_{u}\right|_{k}}=\left\|E^{(k)} \circ T(x)\right\|_{\ell_{k}} .
$$

Also, the sequence $b^{(j)}=\left(b_{n}^{(j)}\right)$ is a Schauder basis for the space $\left|F_{u}\right|_{k}$, where

$$
b_{n}^{(j)}=\left\{\begin{array}{lr}
u_{j}^{-1 / k^{*}} \frac{f_{n+1}}{f_{n}}+u_{j}^{-1 / k^{*}} \sum_{r=j}^{n-1} \frac{f_{n+1}^{2}-f_{n}^{2}}{f_{r} f_{r+1}}, & 0 \leq j \leq n-1 \\
u_{n}^{-1 / k^{*}} \frac{f_{n+1}}{f_{n}}, & j=n \\
0, & j>n .
\end{array}\right.
$$

Proof. We note that $\ell_{k}$ is a $B K$-space for $1 \leq k<\infty$. Further, since $E^{(k)} \circ T$ is a triangle matrix, it follows from Theorem 4.3.2 of [37], $\left|F_{u}\right|_{k}=\left(\ell_{k}\right)_{E^{(k)} \circ \mathrm{T}}$ is a $B K$ space. Since the sequence $\left(e^{(j)}\right)$ is the Schauder basis of the space $\ell_{k}$, it can be written from Theorem 2.3 in [14] that $b^{(j)}=\left(\widetilde{T}_{n}\left(\widetilde{E}^{(k)}\left(e^{(j)}\right)\right)\right)$ is a Schauder basis of the space $\left|F_{u}\right|_{k}$.

Theorem 3.4. Let $1 \leq k<\infty$. Then, the space $\left|F_{u}\right|_{k}$ is isomorphic to the space $\ell_{k}$ that is, $\left|F_{u}\right|_{k} \cong \ell_{k}$.

Proof. To prove the theorem, we should show that there exists a linear bijection between the spaces $\left|F_{u}\right|_{k}$ and $\ell_{k}$ where $1 \leq k<\infty$. Let consider the transformations $T:\left|F_{u}\right|_{k} \rightarrow\left(\ell_{k}\right)_{E^{(k)}}, E^{(k)}:\left(\ell_{k}\right)_{E^{(k)}} \rightarrow \ell_{k}$ given in (1.3) and (1.4). Since the matrices corresponding these transformations are triangles, it can be easily seen that $T$ and $E^{(k)}$ are linear bijections. So, the composite function $E^{(k)} \circ T$ is a linear bijective operator. Furthermore,

$$
\|x\|_{\left|F_{u}\right|_{k}}=\left\|E^{(k)} \circ T(x)\right\|_{\ell_{k}}
$$

i.e., it preserves the norm. So the proof is completed.

In the following theorems, for the simplicity of presentation we take

$$
\xi_{v r}=\left(\frac{f_{v+1}}{f_{v}}+\left(f_{v+1}^{2}-f_{v}^{2}\right) \sum_{j=r}^{v-1} \frac{1}{f_{j} f_{j+1}}\right)
$$


and define

$$
\begin{aligned}
& D_{1}=\left\{\epsilon \in \omega: \sum_{v=r+1}^{\infty} \xi_{v r} \epsilon_{v} \text { exists for all } r\right\}, \\
& D_{2}=\left\{\epsilon \in \omega: \sup _{m}\left(\frac{1}{u_{m}}\left|\epsilon_{m} \frac{f_{m+1}}{f_{m}}\right|^{k^{*}}+\sum_{r=0}^{m-1} \frac{1}{u_{r}}\left|\epsilon_{r} \frac{f_{r+1}}{f_{r}}+\sum_{v=r+1}^{m} \xi_{v r} \epsilon_{v}\right|^{k^{*}}\right)<\infty\right\}, \\
& D_{3}=\left\{\epsilon \in \omega: \sup _{m, r}\left(\left|\epsilon_{m} \frac{f_{m+1}}{f_{m}}\right|+\left|\epsilon_{r} \frac{f_{r+1}}{f_{r}}+\sum_{v=r+1}^{m} \xi_{v r} \epsilon_{v}\right|\right)<\infty\right\}, \\
& D_{4}=\left\{\epsilon \in \omega: \sum_{r=0}^{\infty} \frac{1}{u_{r}}\left(\sum_{v=r+1}^{\infty}\left|\xi_{v r} \epsilon_{v}\right|+\left|\epsilon_{r} \frac{f_{r+1}}{f_{r}}\right|\right)^{k^{*}}<\infty\right\}, \\
& D_{5}=\left\{\epsilon \in \omega: \sup _{r}\left(\sum_{v=r+1}^{\infty}\left|\xi_{v r} \epsilon_{v}\right|+\left|\epsilon_{r} \frac{f_{r+1}}{f_{r}}\right|\right)<\infty\right\} .
\end{aligned}
$$

Theorem 3.5. Let $1<k<\infty$ and $u=\left(u_{n}\right)$ be a sequence of positive numbers. Then,

(i) $\left\{\left|F_{u}\right|\right\}^{\alpha}=D_{5},\left\{\left|F_{u}\right|_{k}\right\}^{\alpha}=D_{4}$;

(ii) $\left\{\left|F_{u}\right|\right\}^{\beta}=D_{1} \cap D_{3},\left\{\left|F_{u}\right|_{k}\right\}^{\beta}=D_{1} \cap D_{2}$;

(iii) $\left\{\left|F_{u}\right|\right\}^{\gamma}=D_{3},\left\{\left|F_{u}\right|_{k}\right\}^{\gamma}=D_{2}$.

Proof. (ii) Let's recall that $\epsilon \in\left\{\left|F_{u}\right|_{k}\right\}^{\beta}$ if and only if $\epsilon x=\left(\epsilon_{n} x_{n}\right) \in c_{s}$ for all $x \in\left|F_{u}\right|_{k}$. By (1.3) and (1.4), it can be seen immediately that

$$
\begin{aligned}
\sum_{v=0}^{m} \epsilon_{v} x_{v} & =\epsilon_{0} x_{0}+\sum_{v=1}^{m} \epsilon_{v}\left(\frac{f_{v+1}}{f_{v}} y_{v}+\left(f_{v+1}^{2}-f_{v}^{2}\right) \sum_{j=0}^{v-1} \frac{y_{j}}{f_{j} f_{j+1}}\right) \\
& =\sum_{r=0}^{m} u_{r}^{-1 / k^{*}} \sum_{v=r}^{m} \epsilon_{v} \frac{f_{v+1}}{f_{v}} z_{r}+\sum_{r=0}^{m-1}\left(\sum_{v=r+1}^{m} \epsilon_{v}\left(f_{v+1}^{2}-f_{v}^{2}\right) \sum_{j=r}^{v-1} \frac{1}{f_{j} f_{j+1}}\right) u_{r}^{-1 / k^{*}} z_{r} \\
& =u_{m}^{-1 / k^{*}} \epsilon_{m} \frac{f_{m+1}}{f_{m}} z_{m}+\sum_{r=0}^{m-1} u_{r}^{-1 / k^{*}}\left(\epsilon_{r} \frac{f_{r+1}}{f_{r}}+\sum_{v=r+1}^{m} \epsilon_{v} \xi_{v r}\right) z_{r} \\
& =\sum_{r=0}^{m} h_{m r} z_{r} \quad\left(y=T(x), z=E^{(k)}(y)\right)
\end{aligned}
$$

where $H=\left(h_{m r}\right)$ is defined by

$$
h_{m r}= \begin{cases}u_{r}^{-1 / k^{*}}\left(\epsilon_{r} \frac{f_{r+1}}{f_{r}}+\sum_{v=r+1}^{m} \epsilon_{v} \xi_{v r}\right), & 0 \leq r \leq m-1, \\ u_{m}^{-1 / k^{*}} \frac{f_{m+1}}{f_{m}} \epsilon_{m}, & r=m \\ 0, & r>m .\end{cases}
$$

Therefore, $\epsilon \in\left\{\left|F_{u}\right|_{k}\right\}^{\beta}$ if and only if $H \in\left(\ell_{k}, c\right)$. Applying Lemma 1.4 to the matrix $H$, we get $\left\{\left|F_{u}\right|_{k}\right\}^{\beta}=D_{1} \cap D_{2}$, which completes the proof.

The proofs of other parts can similarly be proved, so we omit. 
Theorem 3.6. Let $1 \leq k<\infty, A=\left(a_{n v}\right)$ be an infinite matrix of complex numbers for each $n, v \in \mathbb{N}$ and define the matrix $B^{(n)}=\left(b_{m r}^{(n)}\right)$ by

$$
b_{m r}^{(n)}= \begin{cases}a_{n r} \frac{f_{r+1}}{f_{r}}+\sum_{v=r+1}^{m} a_{n v} \xi_{v r}, & 0 \leq r \leq m-1, \\ \frac{f_{m+1}}{f_{m}} a_{n m}, & r=m, \\ 0, & r>m .\end{cases}
$$

Further, let $\bar{B}=\left(\bar{b}_{n v}\right)$ be a matrix given by $\bar{b}_{n v}=\lim _{m} b_{m v}^{(n)}$ and $\widetilde{B}=E^{(k)} \circ T \circ \bar{B}$. Then, $A \in\left(\left|F_{u}\right|,\left|F_{u}\right|_{k}\right)$ if and only if

$$
\begin{gathered}
\sum_{v=r+1}^{\infty} \xi_{v r} a_{n v} \text { exists for all } r \\
\sup _{m, r}\left\{\left|a_{n m} \frac{f_{m+1}}{f_{m}}\right|+\left|a_{n r} \frac{f_{r+1}}{f_{r}}+\sum_{v=r+1}^{m} \xi_{v r} a_{n v}\right|\right\}<\infty, \\
\sup _{r} \sum_{n=0}^{\infty} \frac{1}{u_{r}}\left|\widetilde{b}_{n r}\right|^{k}<\infty,
\end{gathered}
$$

If $A \in\left(\left|F_{u}\right|,\left|F_{u}\right|_{k}\right)$, then $A$ is a bounded linear operator,

$$
\|A\|_{\left(\left|F_{u}\right|,\left|F_{u}\right|_{k}\right)}=\|\widetilde{B}\|_{\left(l, l_{k}\right)}
$$

and

$$
\|A\|_{\chi}=\lim _{v \rightarrow \infty}\left\{\sup _{r} \sum_{n=v+1}^{\infty} \frac{1}{u_{r}}\left|\widetilde{b}_{n r}\right|^{k}\right\}^{\frac{1}{k}} .
$$

Proof. $A \in\left(\left|F_{u}\right|,\left|F_{u}\right|_{k}\right)$ if and only if $\left(a_{n v}\right)_{v=0}^{\infty} \in\left\{\left|F_{u}\right|\right\}^{\beta}$ and $A(x) \in\left|F_{u}\right|_{k}$ for all $x \in\left|F_{u}\right|$. Now, it can be easily seen from Theorem 3.5, $\left(a_{n v}\right)_{v=0}^{\infty} \in\left\{\left|F_{u}\right|\right\}^{\beta}$ if and only if (3.1) and (3.2) hold. On the other hand, if a matrix $R=\left(r_{n v}\right) \in(\ell, c)$, then the series $R_{n}(x)=\sum_{v=0}^{\infty} r_{n v} x_{v}$ converges uniformly in $n$, because, the remaining term of the series tends to zero uniformly in $n$, since

$$
\left|\sum_{v=m}^{\infty} r_{n v} x_{v}\right| \leq \sup _{v}\left|r_{n v}\right| \sum_{v=m}^{\infty}\left|x_{v}\right| \rightarrow 0, \quad m \rightarrow \infty .
$$

So we obtain

$$
\lim _{n} R_{n}(x)=\sum_{v=0}^{\infty} \lim _{n} r_{n v} x_{v} .
$$

Using (1.3), (1.4) and (3.4) it can be written that

$$
A_{n}(x)=\lim _{m} \sum_{k=0}^{m} a_{n k} x_{k}=\lim _{m} \sum_{r=0}^{m} b_{m r}^{(n)} z_{r}=\sum_{r=0}^{\infty} \bar{b}_{n r} z_{r} .
$$


Besides, according to Theorem 3.4, since $\left|F_{u}\right|_{k} \cong \ell_{k}$ for $1 \leq k<\infty$, it follows that $A(x) \in\left|F_{u}\right|_{k}$ for all $x \in\left|F_{u}\right|$ if and only if $\bar{B} \in\left(\ell,\left|F_{u}\right|_{k}\right)$, or equivalently, since $\left|F_{u}\right|_{k}=\left(\ell_{k}\right)_{E^{(k)} \circ T}, \widetilde{B} \in\left(\ell, \ell_{k}\right)$. Also, it is clear that the terms of matrix $\widetilde{B}$ can be expressed as

$$
\begin{aligned}
& \hat{b}_{n r}=\sum_{v=0}^{n} t_{n v} \bar{b}_{v r}=\frac{f_{n}}{f_{n+1}} \bar{b}_{n r}+\sum_{v=0}^{n-1} \frac{f_{n}^{2}-f_{n+1}^{2}}{f_{n} f_{n+1}} \bar{b}_{v r}, \\
& \tilde{b}_{n r}=u_{r}^{1 / k^{*}}\left(\hat{b}_{n r}-\hat{b}_{n-1, r}\right), \quad n \geq 1 \text { and } \widetilde{b}_{0 r}=\bar{b}_{0 r} .
\end{aligned}
$$

Hence, applying Lemma 1.3 to the matrix $\widetilde{B}$, we have (3.3), which completes the first part of the proof.

Also, if $A \in\left(\left|F_{u}\right|,\left|F_{u}\right|_{k}\right)$, then, since the spaces $\left|F_{u}\right|_{k}$ and $\left|F_{u}\right|$ are $B K$-spaces, it is a bounded operator. In order to determine the operator norm of $A$, consider the isomorphisms $T:\left|F_{u}\right|_{k} \rightarrow\left(\ell_{k}\right)_{E^{(k)}}, E^{(k)}:\left(\ell_{k}\right)_{E^{(k)}} \rightarrow \ell_{k}$ defined as in Theorem 3.4. Then, it is easy to see that $A=\widetilde{T} \circ \widetilde{E}^{(k)} \circ \widetilde{B} \circ E^{(1)} \circ T$ and so,

$$
\begin{aligned}
\|A\|_{\left(\left|F_{u}\right|,\left|F_{u}\right|_{k}\right)} & =\sup _{x \neq 0} \frac{\|A(x)\|_{\left|F_{u}\right|_{k}}}{\|x\|_{\left|F_{u}\right|}}=\sup _{x \neq 0} \frac{\left\|\widetilde{T} \circ \widetilde{E}^{(k)} \circ \widetilde{B} \circ E^{(1)} \circ T(x)\right\|_{\left|F_{u}\right|_{k}}}{\|x\|_{\left|F_{u}\right|}} \\
& =\sup _{z \neq 0} \frac{\|\widetilde{B}(z)\|_{\ell_{k}}}{\|z\|_{\ell}}=\|\widetilde{B}\|_{\left(\ell, \ell_{k}\right)}\left(z=E^{(1)} \circ T(x)\right) .
\end{aligned}
$$

Finally, assume that $Q$ is a unique ball in $\left|F_{u}\right|$. Since $E^{(k)} \circ T \circ A Q=\widetilde{B} \circ E^{(1)} \circ T Q$, we get that

$$
\begin{aligned}
\|A\|_{\chi}=\chi(A Q) & =\chi\left(E^{(k)} \circ T \circ A Q\right)=\chi\left(\widetilde{B} \circ E^{(1)} \circ T Q\right) \\
& =\lim _{v \rightarrow \infty}\left(\sup _{z \in E^{(1)}(T(Q))}\left\|\left(I-P_{v}\right)(\widetilde{B}(z))\right\|\right) \\
& =\lim _{v \rightarrow \infty}\left\{\sup _{r}\left(\sum_{n=v+1}^{\infty} \frac{1}{u_{r}}\left|\widetilde{b}_{n r}\right|\right)^{k}\right\}^{\frac{1}{k}} .
\end{aligned}
$$

This completes the proof.

By Theorem 3.6 and Lemma 2.2, the compact operators in this class are characterized as follows.

Corollary 3.1. Under the hypothesis of Theorem 3.6

$$
A \in\left(\left|F_{u}\right|,\left|F_{u}\right|_{k}\right) \text { is compact if and only if } \lim _{v \rightarrow \infty}\left\{\sup _{r}\left(\sum_{n=v+1}^{\infty} \frac{1}{u_{r}}\left|\widetilde{b}_{n r}\right|\right)^{k}\right\}^{\frac{1}{k}}=0 \text {. }
$$

Theorem 3.7. Let $1<k<\infty, A=\left(a_{n v}\right)$ be an infinite matrix of complex numbers for all $n, v \in \mathbb{N}$ and $B^{(n)}=\left(b_{m v}^{(n)}\right)$ be as in Theorem 3.6. Besides, define $\bar{H}=\left(h_{n v}\right)$ 
by $\bar{h}_{n v}=\lim _{m} u_{v}^{-1 / k^{*}} b_{m v}^{(n)}$ and $\widetilde{H}=E^{(1)} \circ T \circ \bar{H}$. Then, $A \in\left(\left|F_{u}\right|_{k},\left|F_{u}\right|\right)$ if and only if

$$
\begin{gathered}
\sum_{v=r+1}^{\infty} \xi_{v r} a_{n v} \text { exist for all } r, \\
\sup _{m}\left\{\frac{1}{u_{m}}\left|a_{n m} \frac{f_{m+1}}{f_{m}}\right|^{k^{*}}+\sum_{r=0}^{m-1} \frac{1}{u_{r}}\left|a_{n r} \frac{f_{r+1}}{f_{r}}+\sum_{v=r+1}^{m} \xi_{v r} a_{n v}\right|^{k^{*}}\right\}<\infty, \\
\sum_{r=0}^{\infty}\left(\sum_{n=0}^{\infty}\left|\widetilde{h}_{n r}\right|\right)^{k^{*}}<\infty .
\end{gathered}
$$

Moreover, if $A \in\left(\left|F_{u}\right|_{k},\left|F_{u}\right|\right)$, then $A$ is a bounded linear operator,

$$
\|A\|_{\left(\left|F_{u}\right|_{k},\left|F_{u}\right|\right)}=\|\widetilde{H}\|_{\left(\ell_{k}, \ell\right)}
$$

and

$$
\|A\|_{\chi}=\frac{1}{\xi} \lim _{v \rightarrow \infty}\left\{\sum_{r=0}^{\infty}\left(\sum_{n=v+1}^{\infty}\left|\widetilde{h}_{n r}\right|\right)^{k^{*}}\right\}^{\frac{1}{k^{*}}}
$$

where $1 \leq \xi \leq 4$.

Proof. $A \in\left(\left|F_{u}\right|_{k},\left|F_{u}\right|\right)$ if and only if $A_{n}=\left(a_{n v}\right)_{v=0}^{\infty} \in\left\{\left|F_{u}\right|_{k}\right\}^{\beta}$ and $A(x) \in\left|F_{u}\right|$ where $x \in\left|F_{u}\right|_{k}$. By Theorem 3.5, it can be easily seen that $A_{n} \in\left\{\left|F_{u}\right|_{k}\right\}^{\beta}$ if and only if (3.5) and (3.6) hold. Also, if any matrix $R=\left(r_{n v}\right) \in\left(\ell_{k}, c\right)$, then the series $R_{n}(x)=\sum_{v=0}^{\infty} r_{n v} x_{v}$ converges uniformly in $n$. Because, the remaining term of the series tends to zero uniformly in $n$, since

$$
\left|\sum_{v=m}^{\infty} r_{n v} x_{v}\right| \leq\left(\sum_{v=m}^{\infty}\left|r_{n v}\right|^{k^{*}}\right)^{\frac{1}{k^{*}}}\left(\sum_{v=m}^{\infty}\left|x_{v}\right|^{k}\right)^{\frac{1}{k}} \rightarrow 0, \quad m \rightarrow \infty
$$

and so, it can be written that

$$
\lim _{n} R_{n}(x)=\sum_{v=0}^{\infty} \lim _{n} r_{n v} x_{v}
$$

Then, using (3.8), with a few calculations, we get

$$
A_{n}(x)=\lim _{m} \sum_{k=0}^{m} a_{n k} x_{k}=\lim _{m} \sum_{r=0}^{m} u_{r}^{-1 / k^{*}} b_{m r}^{(n)} z_{r}=\sum_{r=0}^{\infty} \bar{h}_{n r} z_{r}
$$

Since $\left|F_{u}\right|_{k} \cong \ell_{k}$ for $1 \leq k<\infty$, by the Theorem 3.4, then, $A(x) \in\left|F_{u}\right|$ for every $x \in\left|F_{u}\right|_{k}$ if and only if $\bar{H}(z) \in\left|F_{u}\right|$, i.e., $\widetilde{H}(z)=E^{(1)} \circ T \circ \bar{H}(z) \in \ell$ for every $z \in \ell_{k}$, where $z=E^{(k)} \circ T(x)$. This means that $\widetilde{H} \in\left(\ell_{k}, \ell\right)$. Thus applying Lemma 1.2 to the matrix $\widetilde{H}$, we get (3.7). This completes the proof of first part.

Since $\left|F_{u}\right|_{k}$ is $B K$-spaces for every $k \geq 1, A$ is a bounded operator by Theorem 4.2 .8 of [37]. 
Additionally, as Theorem 3.4, it can be written that $A=\widetilde{T} \circ \widetilde{E}^{(1)} \circ \widetilde{H} \circ E^{(k)} \circ T$ and so,

$$
\begin{aligned}
\|A\|_{\left(\left|F_{u}\right|_{k},\left|F_{u}\right|\right)} & =\sup _{x \neq 0} \frac{\|A(x)\|_{\left|F_{u}\right|}}{\|x\|_{\left|F_{u}\right|_{k}}}=\sup _{x \neq 0} \frac{\left\|\widetilde{H} \circ E^{(k)} \circ T(x)\right\|_{\ell}}{\left\|E^{(k)} \circ T(x)\right\|_{\ell_{k}}} \\
& =\sup _{z \neq 0} \frac{\|\widetilde{H}(z)\|_{\ell}}{\|z\|_{\ell_{k}}}=\|\widetilde{H}\|_{\left(\ell_{k}, \ell\right)} .
\end{aligned}
$$

Finally, let $Q=S_{\left|F_{u}\right|}$. Since $E^{(1)} \circ T \circ A Q=\widetilde{H} \circ E^{(k)} \circ T Q$, it follows by Lemma 2.1, Lemma 2.3 and Lemma 1.2 that

$$
\begin{aligned}
\|A\|_{\chi} & =\chi(A Q)=\chi\left(E^{(1)} \circ T \circ A Q\right)=\chi\left(\widetilde{H} \circ E^{(k)} \circ T Q\right) \\
& =\lim _{v \rightarrow \infty}\left(\sup _{z \in E^{(k)}(T(Q))}\left\|\left(I-P_{v}\right)(\widetilde{H}(z))\right\|_{\ell_{k}}\right) \\
& =\frac{1}{\xi} \lim _{v \rightarrow \infty}\left\{\sum_{r=0}^{\infty}\left(\sum_{n=v+1}^{\infty}\left|\widetilde{h}_{n r}\right|\right)^{k^{*}}\right\}^{\frac{1}{k^{*}}},
\end{aligned}
$$

which completes the proof.

Also, the compact operators can immediately be characterized by Lemma 2.2 and Theorem 3.7 as follows.

Corollary 3.2. Under the conditions of Theorem 3.7

$$
A \in \mathcal{C}\left(\left|F_{u}\right|_{k},\left|F_{u}\right|\right) \Leftrightarrow \frac{1}{\xi} \lim _{v \rightarrow \infty}\left\{\sum_{r=0}^{\infty}\left(\sum_{n=v+1}^{\infty}\left|\widetilde{h}_{n r}\right|\right)^{k^{*}}\right\}^{\frac{1}{k^{*}}}=0 .
$$

\section{REFERENCES}

[1] B. Altay and F. Başar, The fine spectrum and the matrix domain of the difference operator $\Delta$ on the sequence space $\ell_{p}(0<p<1)$, Commun. Math. Anal. 2(2) (2007), 1-11.

[2] B. Altay and F. Başar, Some paranormed Riesz sequence spaces of non-absolute type, Southeast Asian Bull. Math. 30(5) (2006), 591-608.

[3] B. Altay, F. Başar and M. Mursaleen, On the Euler sequence spaces which include the spaces $l_{p}$ and $l_{\infty}$ I, Inform. Sci. 176(10) (2005), 1450-1462.

[4] F. Başar and N. L. Braha, Euler-Cesàro difference spaces of bounded, convergent and null sequences, Tamkang J. Math. 47(4) (2016), 405-420.

[5] F. Başar, Summability Theory and Its Applications, Bentham Science Publishers, e-books, Monographs, Istanbul, 2012.

[6] F. Başar and B. Altay, On the space of sequences of p-bounded variation and related matrix map- pings, (English, Ukrainian summary) Ukrain. Mat. Zh. 55(1) (2003), 108-118, reprinted in Ukrainian Math. J. 55(1) (2003), 136-147.

[7] M. Başarır, F. Başar and E. E. Kara, On the spaces of Fibonacci difference absolutely p-summable, null and convergent sequences, Sarajevo J. Math. 12(25) (2016), 167-182.

[8] J. Boos and P. Cass, Classical and Modern Methods in Summability, Oxford University Press, New York, 2000. 
[9] H. Bor, On summability factors of infinite series, Tamkang J. Math. 16(1) (1985), 13-20.

[10] H. Bor and B. Thorpe, On some absolute summability methods, Analysis 7(2) (1987), 145-152.

[11] T. M. FLett, On an extension of absolute summability and some theorems of Littlewood and Paley, Proc. Lond. Math. Soc. 7 (1957), 113-141.

[12] G. C. Hazar and M. A. Sarıöl, Compact and matrix operators on the space $|C,-1|_{k}$, J. Comput. Anal. Appl. 25 (2018), 1014-1024.

[13] G. C. Hazar and F. Gökçe, Characterizations of matrix transformatios on the series spaces derived by absolute factorable summability, Developments in Science and Engineering (2016), 411-426.

[14] A. M. Jarrah and E. Malkowsky, Ordinary absolute and strong summability and matrix transformations, Filomat 17 (2003), 59-78.

[15] E. E. Kara, Some topological and geometrical properties of new Banach sequence spaces, J. Inequal. Appl. 2013(1) (38) (2013), 16 pages.

[16] E. E. Kara and M. Ilkhan, On some Banach sequence spaces derived by a new band matrix, Br. J. Math. Comput. Sci. 9(2) (2015), 141-159.

[17] E. E. Kara and M. Ilkhan, Some properties of generalized Fibonacci sequence spaces, Linear Multilinear Algebra 64(11) (2016), 2208-2223.

[18] V. Karakaya, A. K. Noman and H. Polat, On paranormed $\lambda$-sequence spaces of non-absolute type, Math. Comput. Modelling 54(5) (2011), 1473-1480.

[19] T. Koshy, Fibonacci and Lucas Numbers with Applications, John Wiley and Sons, New York, 2011.

[20] I. J. Maddox, Elements of Functinal Analysis, Cambridge University Press, London, New York, 1970.

[21] E. Malkowsky and V. Rakocević, On matrix domains of triangles, Appl. Math. Comput. 189(2) (2007), 1146-1163.

[22] E. Malkowsky and V. Rakocević, An introduction into the theory of sequence space and measures of noncompactness, Zb. Rad. (Beogr.) 9(17) (2000), 143-234.

[23] M. R. Mehdi, Summability factors for generalized absolute summability I, Proc. Lond. Math. Soc. 3(1) (1960), 180-200.

[24] R. N. Mohapatra and M. A. Sarıöl, On matrix operators on the series spaces $\left|\bar{N}_{p}^{\theta}\right|_{k}$, Ukrain Mat. Zh. 69(11) (2017), 1524-1533.

[25] M. Mursaleen, F. Başar and B. Altay, On the Euler sequence spaces which include the spaces $l_{p}$ and $l_{\infty}$ II, Nonlinear Anal. 65(3) (2006), 707-717.

[26] C. Orhan and M. A. Sarıöl, On absolute weighted mean summability, Rocky Mountain J. Math. 23(3) (1993), 1091-1097.

[27] V. Rakocević, Measures of noncompactness and some applications, Filomat 12(2) (1998), 87-120.

[28] M. A. Sarıöl, Spaces of series summable by absolute Cesàro and matrix operators, Communications in Mathematics and Applications 7(1) (2016), 11-22.

[29] M. A. Sarıöl, Extension of Mazhar's theorem on summability factors, Kuwait J. Sci. 42(3) (2015), 28-35.

[30] M. A. Sarıöl, Matrix transformations on fields of absolute weighted mean summability, Studia Sci. Math. Hungar. 48(3) (2011), 331-341.

[31] M. A. Sarıöl, Necessary and sufficient conditions for the equivalence of the summability methods $\left|\bar{N}, p_{n}\right|_{k}$ and $|C, 1|_{k}$, Indian J. Pure Appl. Math. 22(6) (1991), 483-489.

[32] M. A. Sarıöl, On the local properties of factored Fourier series, Appl. Math. Comput. 216(11) (2010), 3386-3390.

[33] M. A. Sarıöl, On two absolute Riesz summability factors of infinite series, Proc. Amer. Math. Soc. 118(2) (1993), 485-488.

[34] M. A. Sarıöl, On absolute weighted mean summability methods, Proc. Amer. Math. Soc. 115(1) (1992), 157-160. 
[35] M. Stieglitz and H. Tietz, Matrix transformationen von Folgenraumen. Eine Ergebnisübersicht, Math. Z. 154(1) (1977), 1-16.

[36] W. T. Sulaiman, On summability factors of infinite series, Proc. Amer. Math. Soc. 115 (1992), 313-317.

[37] A. Wilansky, Summability Through Functional Analysis, Mathematics Studies 85, North Holland, Amsterdam, 1984.

${ }^{1}$ Department of MAThematics, UNIVERsity OF PAMUKKALE

Email address: fgokce@pau.edu.tr

Email address: msarigol@pau.edu.tr 